Pacific Journal of Mathematics

EXPECTATIONS IN SEMIFINITE ALGEBRAS 


\title{
EXPECTATIONS IN SEMIFINITE ALGEBRAS
}

\author{
S. K. BERBERIAN
}

Every semifinite von Neumann algebra $A$ possesses an expectation घ: $A \rightarrow W$, where $W$ is a commutative von Neumann subalgebra of $A$ containing the center of $A$, and where $\measuredangle$ extends the trace of a "large" finite subalgebra of $A$. An $A W^{*}$-algebraic proof yields applications to the embedding of semifinite $A W^{*}$-algebras in algebras of type $\mathbf{I}$.

1. Uniform algebras. An algebra of type I may be studied by decomposing it into homogeneous algebras. In an analogous way, we propose to study semifinite algebras via their decompositions into uniform algebras.

Definition [2, p. 242, Exer. 5]. An $A W^{*}$-algebra is said to be uniform if it contains an orthogonal family of equivalent finite projections with supremum 1. (The definition of homogeneous algebra is obtained by replacing "finite" by "abelian".)

LEMMA 1. Every semifinite $A W^{*}$-algebra is the $C^{*}$-sum of a family of uniform algebras.

Proof. Since finite algebras are trivially uniform, one can suppose the given algebra $A$ to be properly infinite. Let $\left(e_{i}\right)_{i \in I}$ be a maximal orthogonal family of pairwise equivalent finite projections; since $A$ is infinite, one can suppose the index set $I$ to be infinite. Then there exist a nonzero central projection $h$ of $A$ and an orthogonal family of projections $\left(f_{i}\right)_{i \in I}$ such that $h=\sup f_{i}$ and $f_{i} \sim h e_{i}$ for all $i \in I$ [1, p. 102, Prop. 2]. This shows that the algebra $h A$ is uniform, and an exhaustion by Zorn's lemma completes the proof.

2. Matrix units. A uniform von Neumann algebra $A$ may be regarded as a tensor product $A=D \otimes L(H)$ with $D$ finite and $L(H)$ the algebra of all bounded operators on a Hilbert space $H$ [2, p. 25, Prop. 5]. There is no analogous theory of tensor product for $A W^{*}$-algebras, but an effective substitute is to pursue the discussion of "matrix units" in $[4, \S 5]$.

Let $A$ be an $A W^{*}$-algebra, with center $Z$, containing an orthogonal family $\left(e_{l}\right)_{i \in I}$ of pairwise equivalent projections with sup $e_{i}=1$. As in [4, §5] construct a family of elements $e_{i j} \in e_{l} A e_{j}(i, j \in I)$ such that $e_{l l}=e_{l}$, $e_{i j}^{*}=e_{l l}, e_{i j} e_{j k}=e_{i k}$ and $e_{l j} e_{m k}=0$ for $j \neq m$. In particular, $e_{\imath j} e_{i j}^{*}=e_{\imath}$ 
and $e_{i j}^{*} e_{i j}=e_{j}$, thus $e_{i j}$ is a partial isometry effecting the equivalence $e_{i} \sim e_{j}$. Let

$$
S=\left\{e_{i j}: i, j \in I\right\}, \quad T=\left\{e_{i}: i \in I\right\}
$$

and let

$$
D=S^{\prime}, \quad W=T^{\prime \prime}
$$

be the commutant and bicommutant, respectively, of these sets in $A ; D$ and $W$ are $A W^{*}$-subalgebras of $A$ with $D=D^{\prime \prime}, W=W^{\prime \prime}[1$, p. 23, Prop. 8]. Since $T$ is a commutative set, $W$ is a commutative algebra; from $W \subset W^{\prime}$ we see that $W^{\prime}$ has center $W$, thus the $e_{i}$ are orthogonal central projections in $W^{\prime}$ with supremum 1 , consequently $W^{\prime}=\bigoplus e_{i} W^{\prime}[1$, p. 53, Prop. 2]. If $x_{i} \in e_{i} W^{\prime}$ for all $i \in I$ and sup $\left\|x_{i}\right\|<\infty$, we write $\oplus x_{i}$ for the unique element $x \in W^{\prime}$ such that $e_{i} x=x_{i}$ for all $i$. Since $T \subset S$, one has

$$
D=S^{\prime} \subset T^{\prime}=T^{\prime \prime \prime}=W^{\prime},
$$

thus $Z \subset W \subset D^{\prime}$. The center of $D$ is $D \cap D^{\prime}=Z$ [4, Lemma 14].

For each $i \in I$, the mapping $d \mapsto d e_{i}$ is a $*$-isomorphism $D \rightarrow e_{i} A e_{i}[4$, Lemma 12], consequently $\left\|d e_{i}\right\|=\|d\|$ for all $d \in D$ and $i \in I[3,1.3 .8$ and 1.8.1]. Moreover [4, Lemma 13],

$$
e_{i} A e_{j}=D e_{i j} \quad(i, j \in I)
$$

the mapping $d \mapsto d e_{i j}$ is an isomorphism of Banach spaces $D \rightarrow e_{i} A e_{j}$, since

$$
\left\|d e_{i j}\right\|^{2}=\left\|\left(d e_{i j}\right)\left(d e_{i j}\right) *\right\|=\left\|d d^{*} e_{i}\right\|=\left\|d d^{*}\right\|=\|d\|^{2}
$$

In particular, for each element $a \in A$ there exists a unique family $\left(a_{i j}\right)$ of elements of $D$ determined by the relations

$$
e_{i} a e_{j}=a_{i j} e_{i j} \quad(i, j \in I)
$$

one calls $\left(a_{i j}\right)$ the "matrix" of $a$ relative to the matrix units $e_{i j}$. One has

$$
\left\|a_{i j}\right\| \leq\|a\| \quad(i, j \in I)
$$

because $\left\|a_{i j}\right\|=\left\|a_{i j} e_{i j}\right\|=\left\|e_{i} a e_{j}\right\|$.

From $D \subset W^{\prime}$ we see that $e_{i} D \subset e_{t} W^{\prime}=e_{i} W^{\prime} e_{l} \subset e_{i} A e_{i}=e_{i} D$, thus $e_{i} W^{\prime}=e_{l} D$; therefore $W^{\prime}=\bigoplus e_{i} D=\oplus e_{i} A e_{i}$. 
LEMMA 2. With the preceding notations,

$$
\begin{aligned}
D^{\prime} & =\left\{a \in A: e_{i} a e_{j} \in Z e_{i j} \text { for all } i, j\right\}, \\
W^{\prime} & =\bigoplus e_{i} W^{\prime}=\bigoplus e_{\imath} D=\bigoplus e_{\imath} A e_{\imath} \\
& =\left\{a \in A: e_{i} a e_{j}=0 \text { whenever } i \neq j\right\},
\end{aligned}
$$

The algebra $D^{\prime}$ is homogeneous, with center $Z$.

Proof. Let $a \in A$ and write $e_{\imath} a e_{J}=a_{i j} e_{\imath j}$ as in (1).

(3) If $d \in D=S^{\prime}$ then $d$ commutes with every $e_{i J}$, therefore

$$
e_{i}(a d-d a) e_{j}=\left(a_{t j} d-d a_{i j}\right) e_{i j} \text {. }
$$

This expression is 0 if and only if $a_{i j} d-d a_{i j}=0$; thus $a \in D^{\prime}$ if and only if $a_{\imath j} \in D \cap D^{\prime}=Z$ for all $i, j$.

(4) The formulas $W^{\prime}=\bigoplus e_{i} W^{\prime}=\oplus e_{l} D=\oplus e_{i} A e_{l}$ are noted above. For all $i, j, k$ one has

$$
\begin{aligned}
e_{i}\left(a e_{k}-e_{k} a\right) e_{j} & =\delta_{j k} a_{i k} e_{i k}-\delta_{i k} a_{k j} e_{k J} \\
& =\delta_{j k} a_{\imath j} e_{i j}-\delta_{\imath k} a_{i j} e_{i j}=\left(\delta_{j k}-\delta_{i k}\right) a_{i j} e_{i j},
\end{aligned}
$$

which is 0 whenever $i=j$. One has $a \in W^{\prime}=T^{\prime}$ if and only if this expression is 0 for all $i, j, k$. If $a \in W^{\prime}$ and $i \neq j$ then $a_{i j}=0$ (take $k=j$ ); on the other hand if $a_{i j}=0$ whenever $i \neq j$, then the expression is 0 for all $i, j, k$, so $a \in W^{\prime}$. Thus $W^{\prime}=\left\{a \in A: e_{i} a e_{j}=0\right.$ for $\left.i \neq j\right\}$.

(5), (6) From (3) we have $e_{i} Z=e_{i} D^{\prime} e_{i}$; since $e_{i} \in W \subset D^{\prime}$, this shows that $e_{l} Z$ is an $A W^{*}$-algebra, and $Z \subset W$ yields $\oplus e_{i} Z \subset \oplus e_{i} W=$ $W$. Obviously $W \subset D^{\prime} \cap W^{\prime}$. If $a \in D^{\prime} \cap W^{\prime}$ then $a=\oplus e_{i} a$ by (4), and $e_{l} a=e_{i} a e_{i}=e_{i} a_{i i}$ with $a_{i i} \in Z$ by (3), thus $a \in \oplus e_{i} Z$. Summarizing, we have $\bigoplus e_{\imath} Z \subset W \subset D^{\prime} \cap W^{\prime} \subset \bigoplus e_{i} Z$, whence equality throughout.

(7) Citing (6), $D \cap W=D \cap D^{\prime} \cap W^{\prime}=Z \cap W^{\prime}=Z$.

Finally, $e_{i j} \in S \subset S^{\prime \prime}=D^{\prime}$ for all $i, j$; this shows that the projections $e_{i}$ are equivalent in $D^{\prime}$. By (3), $e_{i} D^{\prime} e_{i}=Z e_{i}$ is commutative, so the $e_{i}$ are abelian projections in $D^{\prime}$. Thus $D^{\prime}$ is homogeneous, with center $D^{\prime} \cap D^{\prime \prime}=$ $D^{\prime} \cap D=Z$. 
3. Semifinite algebras. The foregoing results on matrix units yield a structure theorem for semifinite algebras; we first review some definitions needed for its statement.

Let $A$ be an $A W^{*}$-algebra, $A_{p}$ its projection lattice, $A_{h}$ the ordered linear space of hermitian elements of $A$ with the set of elements $x^{*} x$ as positive cone; $A$ is said to be normal [15] if $A_{p}$ is monotonely embedded in $A_{h}$, that is, whenever $\left(f_{\alpha}\right)$ is an increasingly directed family of projections with supremum $f$ in $A_{p}$, then $f$ is also the supremum of the family in $A_{h}$ (briefly, $f_{\alpha} \uparrow f$ in $A_{p}$ implies $f_{\alpha} \uparrow f$ in $A_{h}$ ). Every finite $A W^{*}$-algebra is normal [15, Th. 4], as is every $A W^{*}$-algebra that acts faithfully on a separable Hilbert space [16, Cor. 3.4]. (It is not known if there exists a non-normal $A W^{*}$-algebra.) Every von Neumann algebra is normal, hence so is every $W^{*}$-algebra. A positive linear mapping $\varphi: A \rightarrow B$ between $A W^{*}$-algebras is said to be normal if $a_{\alpha} \uparrow a$ in $A_{h}$ implies $\varphi\left(a_{\alpha}\right) \uparrow \varphi(a)$ in $B_{h}$, and completely additive on projections (CAP) if $f_{\alpha} \uparrow f$ in $A_{p}$ implies $\varphi\left(f_{\alpha}\right) \uparrow \varphi(f)$ in $B_{h}$. If $A$ is a normal algebra and $\varphi$ is a normal mapping, then $\varphi$ is CAP.

LemMa 3 [10]. If $A$ is a normal $A W^{*}$-algebra, then for every element $x \in A$ the positive linear mapping $a \mapsto x^{*}$ on $A$ is CAP.

Proof. Suppose $f_{\alpha} \uparrow f$ in $A_{p}$ and $x f_{\alpha} x^{*} \leq b \in A_{h}$ for all $\alpha$; we are to show that $x f x^{*} \leq b$. Let $\varepsilon>0$ and let $c=(b+\varepsilon)^{-1 / 2}$. Then

$$
c x f_{\alpha} x^{*} c \leq c b c=b(b+\varepsilon)^{-1} \leq 1,
$$

thus $\left(c x f_{\alpha}\right)\left(c x f_{\alpha}\right)^{*} \leq 1$; this means that $\left\|c x f_{\alpha}\right\| \leq 1$, so $\left(c x f_{\alpha}\right)^{*}\left(c x f_{\alpha}\right) \leq 1$, whence $f_{\alpha}\left(1-x^{*} c^{2} x\right) f_{\alpha} \geq 0$ for all $\alpha$. It follows from normality that $f\left(1-x^{*} c^{2} x\right) f \geq 0\left[10\right.$, Lemma 3], whence $f x^{*} c^{2} x f \leq f \leq 1,\|c x f\| \leq 1$, $c x f x^{*} c \leq 1, x f x^{*} \leq c^{-2}=b+\varepsilon$. Thus $x f x^{*}-b \leq \varepsilon$ for all $\varepsilon>0$, therefore $x f x^{*}-b \leq 0$.

THEOREM 1. Let $A$ be a semifinite $A W^{*}$-algebra with center $Z$. There exist $A W^{*}$-subalgebras $D$ and $W$ of $A$ with the following properties:

(i) $D=D^{\prime \prime}$ and $W=W^{\prime \prime}$ in $A$;

(ii) $D$ is finite, its center is $Z$, and $D^{\prime}$ is of type I with center $Z ; D$ is *-isomorphic to eAe, with e a faithful finite projection of $A$;

(iii) $W$ is commutative, $W=D^{\prime} \cap W^{\prime}$ and $Z=D \cap W$;

(iv) there is a mapping \#: $A \rightarrow W^{\prime}$ that is left and right $W^{\prime}$-linear, positive, faithful, and leaves fixed the elements of $W^{\prime}$; when $A$ is a normal algebra, the mapping \# is CAP.

(v) If $Z$ is a $W^{*}$-algebra then so are $D^{\prime}$ and $W$; if $D$ is a $W^{*}$-algebra, then so is $W^{\prime}$.

(vi) If $A$ is normal and $D$ is a $W^{*}$-algebra, then $A$ is a $W^{*}$-algebra. 
Proof. By Lemma 1 we are reduced to the case that $A$ is uniform; we adopt the notations of Lemma 2, with the $e_{i}$ finite projections of $A$. In particular, $D$ is $*$-isomorphic to $e_{i} A e_{i}$, hence is finite; the rest of (i)-(iii) is clear from Lemma 2.

(v) The formula $W=D^{\prime} \cap W^{\prime}$ means that $W$ coincides with its commutant in $D^{\prime}$ (thus is a maximal abelian subalgebra of $D^{\prime}$ ); if $Z$ is a $W^{*}$-algebra (that is, $*$-isomorphic to a von Neumann algebra) then so is the type I algebra $D^{\prime}$ with center $Z$ [4, Th. 2], hence so is $W$. On the other hand, if $D$ is a $W^{*}$-algebra, then so are the isomorphic algebras $e_{i} D$, hence so is $W^{\prime}$ by formula (4) of Lemma 2; in this case, the center $Z$ of $D$ is also a $W^{*}$-algebra, hence so are $D^{\prime}$ and $W$.

(iv), (vi) If $a \in A$ then $\left\|e_{i} a e_{i}\right\| \leq\|a\|$ for all $i$, so by (4) of Lemma 2 we can define $a^{\#}=\bigoplus e_{i} a e_{i} \in W^{\prime}$. It is clear that $a \mapsto a^{\#}$ is a positive linear mapping $A \rightarrow W^{\prime}$, leaving fixed the elements of $W^{\prime}$ hence having range $W^{\prime}$. If $a \geq 0$ and $a^{\#}=0$, then $\left(e_{i} a^{1 / 2}\right)\left(e_{i} a^{1 / 2}\right)^{*}=e_{i} a e_{i}=0$ for all $i$, whence $a=0$; thus $\#$ is faithful.

If $c \in W^{\prime}=T^{\prime}$ and $a \in A$, then $c$ commutes with every $e_{i}$, thus $e_{i} c a e_{i}=\left(e_{i} c e_{i}\right)\left(e_{i} a e_{i}\right)$ for all $i$; therefore $(c a)^{\#}=c^{\sharp} a^{\#}=c a^{\sharp}$, similarly $(a c)^{\#}=a^{\#} c$.

Finally, suppose $A$ is a normal algebra and $f_{\alpha} \uparrow f$ in $A_{p}$. By Lemma 3, for each $i$ one has $e_{i} f_{\alpha} e_{i} \uparrow e_{i} f e_{i}$ in $A_{h}$, hence in $\left(e_{i} A e_{i}\right)_{h}$; therefore $\bigoplus e_{i} f_{\alpha} e_{l} \uparrow$ $\bigoplus e_{i} f e_{i}$ in $\left(\bigoplus e_{i} A e_{i}\right)_{h}$, that is, $f_{\alpha}^{\#} \uparrow f^{\#}$ in $\left(W^{\prime}\right)_{h}$. Thus \# is CAP. If, in addition, $D$ is a $W^{*}$-algebra, then by (v) so is $W^{\prime}$, therefore $W^{\prime}$ has a separating family of normal positive linear forms; since \# is CAP, it follows that $A$ has a separating family of positive linear forms that are CAP, therefore $A$ is a $W^{*}$-algebra by a theorem of G. K. Pedersen [7].

4. Trace and expectations. Our next objective is to show that, in the notations of Theorem 1, a center-valued trace $\hbar: D \rightarrow Z$ on the finite algebra $D$ is extendible to a trace-like mapping $\xi: A \rightarrow W$ (more precisely, in the terminology of [6], an expectation of $A$ onto $W$ ). If, in addition, the algebra $A$ is normal, then the resulting expectation of $A$ is a normal mapping. All of these hypotheses are fulfilled when $A$ is a semifinite $W^{*}$-algebra. First, we review a result implicit in [12]:

LeMma 4. Let $A$ be a finite $A W^{*}$-algebra with center $Z$, possessing $a$ trace $\xi: A \rightarrow Z$. Then $A$ is monotone complete and the mapping $\sharp$ is normal.

Proof. The hypothesis is that $\sharp$ is a positive $Z$-linear mapping such that $1^{\natural}=1$ and $(a b)^{\natural}=(b a)^{\natural}$ for all $a, b$ in $A$. It follows that $z^{\natural}=z$ for all $z \in Z$. Moreover, $\downarrow$ is faithful: if $a \geq 0$ and $a^{\natural}=0$ then $a=0$ (because 
every nonzero positive element of $A$ majorizes a positive scalar multiple of a simple projection $[1, \S 26])$.

Let $D: A_{p} \rightarrow Z$ be the dimension function $A[1, \mathrm{p} .181$, Th. 1]. By the uniqueness of $D, e^{\natural}=D(e)$ for all projections $e$; since $D$ is completely additive, $\square$ is CAP $[1$, p. 184, Exer. 4]. It follows that for every $x \in A$, the $Z$-linear mapping $a \mapsto\left(x a x^{*}\right)^{\natural}$ is also CAP (cf. the Appendix), thus $曰$ is continuous in the sense of $[12$, p. 316]. Since $\models$ is faithful, it follows that there exists an $A W^{*}$-algebra $B$ of type $\mathrm{I}$, with center $Z$, such that $A$ is an $A W^{*}$-subalgebra of $B$ [12, Th. 3.1], indeed $A=A^{\prime \prime}$ in $B$ [12, Th. 4.4]. Since $B$ is monotone complete [12, Lemma 1.4] and $A=A^{\prime \prime}$ in $B$, it follows that $A$ is monotone complete. (An $A W^{*}$-algebra $A$ is said to be monotone complete if every increasingly directed family in $A_{h}$, majorized by an element of $A_{h}$, has a supremum in $A_{h}$.)

Suppose $a_{\alpha} \uparrow a$ in $A_{h}$; we are to show that $a_{\alpha}^{\natural} \uparrow a^{\natural}$ in $Z_{h}$. Passing to a cofinal set of indices, we can suppose that $\left\|a_{\alpha}\right\|$ is bounded. Viewing $B$ as the algebra of bounded operators on an $A W^{*}$-module over $Z$ [5, Th. 8], $a_{\alpha}$ is strongly convergent to $a$ [12, Lemma 1.4], therefore $a^{\natural}=\lim \inf a_{\alpha}^{\natural}$ in $Z_{h}$ [12, Lemma 4.3]; since the family $\left(a_{\alpha}^{\natural}\right)$ is increasing, $\lim \inf a_{\alpha}^{\natural}=\sup a_{\alpha}^{\natural}$, thus $a_{\alpha}^{\natural} \uparrow a^{\natural}$ in $Z_{h}$.

In Theorem 2 it will be assumed that the finite algebra $D$ of Theorem 1 has a trace, equivalently, that the isomorphic algebra $e A e$ has a trace; the next two lemmas free this hypothesis from its reference to a particular faithful finite projectión $e$.

Lemma 5. If the finite $A W^{*}$-algebra $A$ has a trace, then so does every corner eAe of $A$ and every matrix algebra $M_{n}(A)$ over $A$.

Proof. If $4: A \rightarrow Z$ is the trace of $A$ ( $Z$ the center of $A$ ) and if $r$ is the relative inverse of $e^{\natural}$ in the regular ring of $A[1, \mathrm{p}$. 235], then the trace $e A e \rightarrow e Z$ of $e A e$ is given by the formula $x \mapsto e r x^{\natural}$. Identifying the center of $M_{n}(A)$ with $Z$, the trace of a matrix is defined to be the average of the traces of its diagonal elements.

LEMMA 6. Let $A$ be a semifinite $A W^{*}$-algebra containing a faithful finite projection $f$ such that fAf has a trace. Then for every finite projection e of $A$, eAe has a trace.

Proof. The first step of the proof is to find a nonzero central projection $h$ of $A$ such that $(h e) A(h e)=h e A e$ has a trace. We can suppose $e \neq 0$; then $e A f \neq 0$ (because $f$ is faithful), so there exist nonzero 
subprojections $e_{1} \leq e, f_{1} \leq f$ with $e_{1} \sim f_{1}$. Passing to a subprojection of $e_{1}$, we can suppose that $e_{1}$ is a simple projection in $e A e[1, \S 26]$. The central cover of $e_{1}$ in $e A e$ has the form he with $h$ a central projection of $A[1, \mathrm{p}$. 37, Prop. 4], thus heAe $=M_{n}\left(e_{1} A e_{1}\right)$ for a suitable integer $n$ (the "order" of $e_{1}$ in $e A e$ ). Since $f A f$ has a trace, so does its corner $f_{1} A f_{1}$ (Lemma 5), hence so does the isomorphic algebra $e_{1} A e_{1}$, hence so does the matrix algebra heAe (Lemma 5).

Let $\left(h_{\alpha}\right)$ be a maximal orthogonal family of nonzero central projections of $A$ such that every $h_{\alpha} e A e$ has a trace. Necessarily $\sup h_{\alpha}=1$ (otherwise the preceding argument could be used to contradict maximality); thus $e A e=\oplus h_{\alpha} e A e, e Z=\bigoplus h_{\alpha} e Z$ ( $Z$ the center of $A$ ), and the traces of the $h_{\alpha} e A e$ may be combined to give a trace for $e A e$.

Theorem 2. Let $A$ be a semifinite $A W^{*}$-algebra with center $Z$, and adopt the notations of Theorem 1. Suppose, in addition, that the finite algebra $D$ has a trace $\mathrm{h}: D \rightarrow Z$ (as is the case when $A$ is a $W^{*}$-algebra). Then the trace of $D$ is extendible to a positive linear mapping $4: A \rightarrow W$ with the following properties:

(i) $w^{\natural}=w$ for all $w \in W$;

(ii) $(w a)^{\natural}=w a^{\natural}=a^{\natural} w=(a w)^{\natural}$ for all $a \in A, w \in W$;

(iii) $a \geq 0$ and $a^{\natural}=0$ imply $a=0$;

(iv) $(a d)^{\natural}=(d a)^{\natural}$ for all $a \in A, d \in D$; equivalently, $(\text { uau })^{\natural}=a^{\natural}$ for all $a \in A$ and all unitary $u \in D$;

(v) if $A$ is a normal algebra, then the mapping $\downarrow: A \rightarrow W$ is normal and there exists a type I $A W^{*}$-algebra $B$ with center $Z$ such that $A=A^{\prime \prime}$ in $B$.

Proof. By Lemma 6 and the proof of Theorem 1, we can suppose $A$ to be uniform; we adopt the notations of Lemma 2, with the $e_{l}$ finite projections, and we write $\sharp: A \rightarrow W^{\prime}$ for the mapping defined in the proof of Theorem 1.

Suppose, more generally, that $\varphi: D \rightarrow Z$ is any positive linear mapping. For each $i \in I$ let $\varphi_{i}: e_{i} A e_{i} \rightarrow e_{i} Z$ be the unique (positive, linear) mapping such that $\varphi_{l}\left(e_{i} d\right)=e_{i} \varphi(d)$ (recall that $d \mapsto e_{i} d$ is a $*$-isomorphism $\left.D \rightarrow e_{i} A e_{i}\right)$; then

$$
\left\|\varphi_{i}\left(e_{i} d\right)\right\| \leq\|\varphi(d)\| \leq\|\varphi\|\|d\|=\|\varphi\|\left\|e_{i} d\right\|,
$$

so $\left\|\varphi_{i}\right\| \leq\|\varphi\|$ for all $i$. Define a mapping $\bar{\varphi}: W^{\prime} \rightarrow W$ as follows. By (4) of Lemma 2, every $x \in W^{\prime}$ has the form $x=\bigoplus x_{i}$ with $x_{i} \in e_{l} A e_{i}$ and $\left\|x_{l}\right\|$ bounded; then $\left\|\varphi_{l}\left(x_{l}\right)\right\|$ is bounded and we can define

$$
\bar{\varphi}(x)=\bigoplus \varphi_{t}\left(x_{i}\right) \in \bigoplus e_{i} Z=W
$$

by (5) of Lemma 2. (So to speak, $\bar{\varphi}=\bigoplus \varphi_{l}$.) 
Composing the positive linear mappings $\sharp: A \rightarrow W^{\prime}$ and $\bar{\varphi}: W^{\prime} \rightarrow W$, we obtain a positive linear mapping $\Phi: A \rightarrow W$, where $\Phi(a)=\bigoplus \varphi_{i}\left(e_{i} a e_{i}\right)$ for $a \in A$; thus if $e_{i} a e_{j}=a_{i j} e_{i j}$ as in (1), we have

$$
\Phi(a)=\bigoplus e_{i} \varphi\left(a_{i i}\right)
$$

$\Phi$ extends $\varphi$. $\left\{\right.$ Proof: If $a \in D$ then $e_{i} a e_{i}=a e_{i}$ shows that $a_{i i}=a$ for all $i$, whence $\Phi(a)=\bigoplus e_{i} \varphi(a)=\varphi(a)$.

If $\varphi$ is faithful then so is $\Phi$. \{Proof: If $\varphi$ is faithful then so is every $\varphi_{l}$, therefore so is $\bar{\varphi}$; since \# is also faithful, so is $\Phi=\bar{\varphi} \circ \#$.

If $\varphi$ is $Z$-linear, then each of the mappings $a \mapsto \varphi\left(a_{i i}\right)$ is $Z$-linear and $\Phi$ is both left and right $W$-linear. \{Proof: Clearly every $\varphi_{i}$ is $e_{i} Z$-linear, therefore $\bar{\varphi}$ is both left and right $\oplus e_{i} Z$-linear, that is, $W$-linear. If $z \in Z$ then $z a$ has matrix $\left(z a_{i j}\right)$, whence the $Z$-linearity of the mappings $a \mapsto$ $\varphi\left(a_{i i}\right)$.

If $\varphi$ is normal then so is $\bar{\varphi}$; if, moreover, $A$ is a normal algebra, then the mappings $\Phi$ and $a \mapsto \varphi\left(a_{i i}\right)$ on $A$ are CAP. \{Proof: If $\varphi$ is normal then so is every $\varphi_{i}$, hence so is $\bar{\varphi}=\bigoplus \varphi_{i}$. Suppose in addition that $A$ is normal. If $f_{\alpha} \uparrow f$ in $A_{p}$, then $f_{\alpha}^{\#} \uparrow f^{\#}$ in $\left(W^{\prime}\right)_{h}$ by (iv) of Theorem 1, therefore $\bar{\varphi}\left(f_{\alpha}^{\sharp}\right) \uparrow \bar{\varphi}\left(f^{\sharp}\right)$ in $W_{h}$, that is, $\Phi\left(f_{\alpha}\right) \uparrow \Phi(f)$; thus $\Phi$ is CAP. Also, for each $i$ the mapping $a \mapsto e_{i} a e_{i}=e_{i} a_{i i}$ is CAP (Lemma 3); by virtue of the *-isomorphism $e_{i} D \rightarrow D$ and the normality of $\varphi$, it follows that the mapping $a \mapsto \varphi\left(a_{i i}\right)$ is also CAP.

Assume now that there exists a trace $\xi: D \rightarrow Z$ and let $\downarrow$ play the role of $\varphi$. By the foregoing remarks, the mapping $\curvearrowleft: A \rightarrow W$ defined by the formula

$$
a^{\natural}=\bigoplus e_{i} a_{i l}^{\natural}
$$

is left and right $W$-linear, positive, faithful, and extends the trace of $D$; thus the properties (ii), (iii) are verified, hence so is (i) (because $1^{\natural}=1$ ). If $a \in A$ has matrix $\left(a_{i j}\right)$ and if $u \in D$ is unitary, then $u a u^{*}$ has matrix $\left(u a_{i j} u^{*}\right)$, therefore

$$
\left(u a u^{*}\right)^{\natural}=\bigoplus e_{i}\left(u a_{i i} u^{*}\right)^{\natural}=\bigoplus e_{i} a_{i i}^{\natural}=a^{\natural} .
$$

This is equivalent to the identity $(a d)^{\natural}=(d a)^{\natural}$ since every $d \in D$ is a linear combination of unitary elements of $D[2$, p. 4, Prop. 3].

The trace of $D$ is normal (Lemma 4); if, moreover, $A$ is a normal algebra, the above remarks show that the mappings $\sharp: A \rightarrow W$ and $a \mapsto a_{i i}^{\natural}$ on $A$ are CAP; in particular, $A$ has a family of $Z$-linear mappings $A \rightarrow Z$ that are CAP and separating (for, if $a \geq 0$ and $a_{i i}^{\natural}=0$ for all $i$, then 
$a^{\natural}=0$, therefore $a=0$ ). It then follows from K. Saitô's embedding theorem [9, Th. 2] that there exists a type I $A W^{*}$-algebra $B$ with center $Z$, such that $A=A^{\prime \prime}$ in $B$. By the arguments in the proof of Lemma $4, A$ is monotone complete and the above-mentioned $Z$-linear mappings $A \rightarrow Z$ are normal, therefore so is the mapping $\downarrow: A \rightarrow W$.

The following corollary is due in essence to H. Widom [11, Th. 6.3]:

COROLlary 1. If $A$ is a normal, semifinite $A W^{*}$-algebra containing a faithful finite projection $f$ such that $f A f$ has a trace, then $A$ may be embedded as a bicommutant in a type I algebra with the same center.

Proof. With notation as in Theorem 1, it follows from Lemma 6 that $e A e$ has a trace, hence so does the isomorphic algebra $D$; thus all of the hypotheses of Theorem 2 are fulfilled.

[We remark that the result in [11, Th. 6.3] is stated without assuming normality, but normality figures in the proof $[11$, p. 55 , line 4] via an appeal to the property in Lemma 3 above. The countability hypothesis in [11, Th. 6.3] can be omitted by virtue of Saitô's embedding theory [9, Th. 1].\}

COROLlaRY 2. If, under the hypotheses of Corollary 1, the center of $A$ is a $W^{*}$-algebra, then $A$ is also a $W^{*}$-algebra.

Proof. The type I algebra given by Corollary 1 is also $W^{*}[4$, Th. 2], hence so is its subalgebra $A$.

It is an open question whether every $A W^{*}$-factor of type $\mathrm{II}_{1}$ has a trace; if the answer is yes, then Corollary 2 would imply that every normal $A W^{*}$-factor of type $\mathrm{II}_{\infty}$ is a $W^{*}$-algebra.

Corollary 3 [13, p. 445, Cor.]. Let $A$ be a normal, semifinite $A W^{*}$-algebra whose center $Z$ is a $W^{*}$-algebra. If $A$ has a faithful positive linear form then it is a $W^{*}$-algebra.

Proof. With notations as in Theorem 1 , the finite algebra $D$ also has center $Z$ and has a faithful positive linear form, hence is a $W^{*}$-algebra [14, p. 437, Cor. 7]; therefore $D$ has a trace and Corollary 2 applies.

5. Appendix. The following proposition (stated without proof in [8]) is implicit in the proof of Saitô's embedding theorem [9, Th. 2]; the brief proof given here was communicated to me by Professor Saitô. 
Proposition [8, 1.1.2]. If $A$ is an $A W^{*}$-algebra, $B$ is a commutative $A W^{*}$-algebra, and $\varphi: A \rightarrow B$ is a positive linear mapping that is CAP, then for every $x \in A$ the mapping $a \mapsto \varphi\left(x^{*}\right)$ is also CAP.

Proof. Assuming $f_{\alpha} \downarrow 0$ in $A_{p}$, it will suffice to show that $\varphi\left(x f_{\alpha} x^{*}\right) \downarrow 0$ in $B_{h}$. This is clear if $x$ is unitary, for then $x f_{\alpha} x^{*} \downarrow 0$ in $A_{p}$. In general, $x$ is a linear combination of four unitaries, say $x=\sum_{i=1}^{4} \lambda_{i} u_{i}$. Then

$$
\varphi\left(x f_{\alpha} x^{*}\right)=\sum_{i, j} \lambda_{i} \bar{\lambda}_{j} \varphi\left(u_{i} f_{\alpha} u_{j}^{*}\right)
$$

Writing $|b|=\left(b^{*} b\right)^{1 / 2}$ for $b \in B$, the Cauchy-Schwarz inequality [cf. 5, p. 840] yields

$$
\begin{aligned}
\left|\varphi\left(u_{i} f_{\alpha} u_{j}^{*}\right)\right|^{2} & =\left|\varphi\left(u_{i}\left(u_{j} f_{\alpha}\right) *\right)\right|^{2} \\
& \leq \varphi\left(u_{i} u_{i}^{*}\right) \varphi\left(u_{j} f_{\alpha} u_{j}^{*}\right)=\varphi(1) \varphi\left(u_{j} f_{\alpha} u_{j}^{*}\right)
\end{aligned}
$$

writing $M=\max \left|\lambda_{i} \lambda_{j}\right|$, we thus have

$$
\varphi\left(x f_{\alpha} x^{*}\right) \leq 4 M \varphi(1)^{1 / 2} \sum_{j=1}^{4} \varphi\left(u_{j} f_{\alpha} u_{j}^{*}\right)^{1 / 2},
$$

where $\varphi\left(u_{j} f_{\alpha} u_{j}^{*}\right)^{1 / 2} \downarrow 0$ in $B_{h}$ for each $j$, therefore also $\varphi\left(x f_{\alpha} x^{*}\right) \downarrow 0$.

We remark that for the CAP mappings occurring in Lemmas 3 and 4 (hence in Theorems 1 and 2), the conclusion of the Proposition can be seen directly: in the case of Lemma 3, one notes that $y\left(x f_{\alpha} x^{*}\right) y^{*}=$ $(y x) f_{\alpha}(y x)^{*}$; in the case of Lemma $4,\left(x f_{\alpha} x^{*}\right)^{\natural}=\left(f_{\alpha} x^{*} x f_{\alpha}\right)^{\natural} \leq\|x\|^{2} f_{\alpha}^{\natural}$.

Problems. 1. Is every semifinite $A W^{*}$-algebra normal?

2 . In the notations of Lemma 2 , does every *-automorphism of $D$ extend to a $*$-automorphism of $A$ ?

3. If $A$ is an $A W^{*}$-algebra containing a faithful projection $e$ such that $e A e$ is a $W^{*}$-algebra, does it follow that $A$ is a $W^{*}$-algebra? (The answer is yes if $A$ is normal.)

ACKNOwledgement. This work was done while I was a Senior Visiting Fellow at the Univeristy of Reading. I am deeply indebted to my colleagues J. D. M. Wright and K. Saitô, whose insights and suggestions greatly improved the manuscript. 


\section{REFERENCES}

1. S. K. Berberian, Baer *-Rings, Springer-Verlag, New York, 1972.

2. J. Dixmier, Les algèbres d'opérateurs dans l'espace hilbertien (Algèbres de von Neumann ), $2^{e}$ edn., Gauthier-Villars, Paris, 1969.

3. Les $C^{*}$-algèbres et leurs représentations, $2^{e}$ edn., Gauthier-Villars, Paris, 1969.

4. I. Kaplansky, Algebras of type I, Ann. of Math., (2) 56 (1952), 460-472.

$5 . \quad$ Modules over operator algebras, Amer. J. Math., 75 (1953), 839-858.

6. M. Nakamura and T. Turumaru, Expectations in an operator algebra, Tôhoku Math. J.,

(2) 6 (1954), 182-188.

7. G. K. Pedersen, Operator algebras with weakly closed abelian subalgebras, Bull. London Math. Soc., 4 (1972), 171-175.

8. K. Saitô, On the embedding as a double commutator in a type I $A W^{*}$-algebra, Tôhoku Math. J., (2) 23 (1971), 541-557.

9. __ On the embedding as a double commutator in a type I A $W^{*}$-algebra. II, Tôhoku Math. J., (2) 26 (1974), 333-339.

10. __ On normal $A W^{*}$-algebras, Tôhoku Math. J., (2) 33 (1981), 567-571.

11. H. Widom, Embedding of $A W^{*}$-algebras, Ph.D. Thesis, University of Chicago, 1955.

12. __ Embedding in algebras of type I, Duke Math. J., 23 (1956), 309-324.

13. J. D. M. Wright, On semifinite $A W^{*}$-algebras, Math. Proc. Cambridge Phil. Soc., 74 (1975), 443-445.

14. , On $A W^{*}$-algebras of finite type, J. London Math. Soc., (2) 12 (1976), $431-439$.

15. Normal AW*-algebras, Proc. Royal Soc. Edinburgh, 85A (1980), 137-141.

16. On some problems of Kaplansky in the theory of rings of operators, Math. Z., 172 (1980), 131-141.

Received March 5, 1982 and in revised form April 12, 1982. The author gratefully acknowledges the support of the S.E.R.C. (Grant No. GR/B 60514).

UNIVERSITY OF TEXAS

AUSTIN, TX 78712

AND

UNIVERSITY OF READING

U. K. 



\section{PACIFIC JOURNAL OF MATHEMATICS EDITORS}

DONALD BABBITT (Managing Editor)

University of California

Los Angeles, CA 90024

Hugo Rossi

University of Utah

Salt Lake City, UT 84112

C. C. Moore and Arthur Ogus

University of California

Berkeley, CA 94720
J. DugunduI

Department of Mathematics

University of Southern California

Los Angeles, CA 90089-1113

R. FinN and H. SAmelson

Stanford University

Stanford, CA 94305

\section{ASSOCIATE EDITORS}
R. ARENS
E. F. BECKENBACH
B. H. NeUmaNN
F. WolF
K. YoshidA (1906-1982)

\section{SUPPORTING INSTITUTIONS}

UNIVERSITY OF ARIZONA

UNIVERSITY OF BRITISH COLUMBIA

CALIFORNIA INSTITUTE OF TECHNOLOGY

UNIVERSITY OF CALIFORNIA

MONTANA STATE UNIVERSITY

UNIVERSITY OF NEVADA, RENO

NEW MEXICO STATE UNIVERSITY

OREGON STATE UNIVERSITY
UNIVERSITY OF OREGON

UNIVERSITY OF SOUTHERN CALIFORNIA

STANFORD UNIVERSITY

UNIVERSITY OF HAWAII

UNIVERSITY OF TOKYO

UNIVERSITY OF UTAH

WASHINGTON STATE UNIVERSITY

UNIVERSITY OF WASHINGTON 


\section{Pacific Journal of Mathematics}

\section{Vol. 106, No. $1 \quad$ November, 1983}

John Ballard, Clifford's theorem for algebraic groups and Lie algebras . . . . . 1

Philip Richard Bartick, II and Edwin Duda, Finite-to-one open mappings

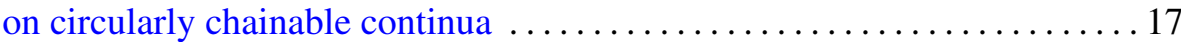

Frank Hayne Beatrous, Jr., $H^{\infty}$-interpolation from a subset of the

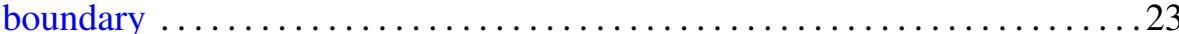

Sterling K. Berberian, Expectations in semifinite algebras ............ 33

Heron S. Collins and Wolfgang Ruess, Weak compactness in spaces of compact operators and of vector-valued functions $\ldots \ldots \ldots \ldots \ldots \ldots . \ldots 45$

David Downing and William O. Ray, Renorming and the theory of

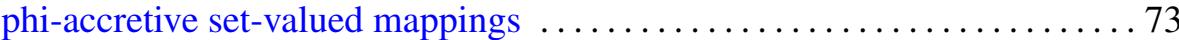

Harvey Charles Greenwald, On the theory of homogeneous Lipschitz

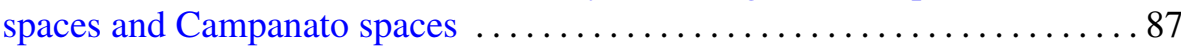

Irwen Valle Guadalupe and Lucio Ladislao Rodriguez, Normal curvature

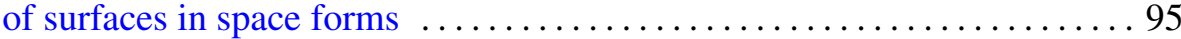

Boju Jiang, On the computations of the Nielsen number ............... 105

William H. Julian, Ray Mines, III and Fred Richman, Alexander

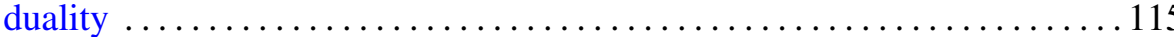

Midori Kobayashi, The connected component of the idèle class group of an

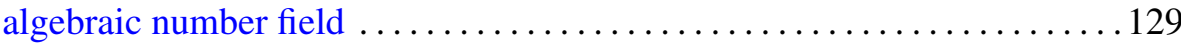

Ib Henning Madsen, Charles B. Thomas and C. Terence C. (Charles)

(Clegg) Wall, Topological spherical space form problem. III.

Dimensional bounds and smoothing

A. Sapounakis, The existence of strong liftings for totally ordered measure

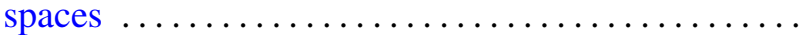

Gary Seitz, The root subgroups for maximal tori in finite groups of Lie type

Laurent Siebenmann and James M. Van Buskirk, Construction of irreducible homology 3-spheres with orientation reversing involution 